\title{
Microcontroller based double beam modulation system for atomic scattering experiments
}

\author{
R W O’Neill ${ }^{1}$, J B Greenwood ${ }^{2}$, M L Gradziel ${ }^{1}$ and I D Williams ${ }^{2}$ \\ ${ }^{1}$ Department of Experimental Physics, National University of Ireland, Maynooth, Maynooth, \\ Co. Kildare, Ireland \\ ${ }^{2}$ Department of Pure and Applied Physics, The Queen's University of Belfast, \\ Belfast BT7 1NN, UK
}

Received 24 January 2001, in final form 13 June 2001, accepted for publication 27 June 2001

Published 2 August 2001

Online at stacks.iop.org/MST/12/1480

\begin{abstract}
Double beam modulation is widely used in atomic collision experiments in the case where the noise arising from each of the beams exceeds the measured signal. A method for minimizing the statistical uncertainty in a measured signal in a given time period is discussed, and a flexible modulation and counting system based on a low cost PIC microcontroller is described. This device is capable of modifying the acquisition parameters in real time during the course of an experimental run. It is shown that typical savings in data acquisition time of approximately $30 \%$ can be achieved using this optimized modulation scheme.
\end{abstract}

Keywords: electron-ion, beam modulation, microcontroller, statistical optimization

\section{Introduction}

In atomic collision experiments it is often the case that a small experimental signal is swamped by extraneous noise. In a typical case where a fast projectile beam of electrons or ions collides with a thermal neutral beam target it is common practice to modulate the gas jet providing the target beam. In this way, the signal resulting from projectile interaction with the target is separated from that due to projectile interaction with the background gas. Phase sensitive detection or gated particle counting techniques may then be used to enhance the signal to noise ratio.

In experiments where two beams interact and both give rise to backgrounds, it is generally necessary to modulate both beams in order to extract useful signal from the background noise. Double beam modulation is inevitable in the specific case of two fast charged beams interacting, since space charge limits the maximum density of each beam to approximately $10^{6} \mathrm{~cm}^{-3}$. This is comparable to the density of the background gas, even under ultra-high vacuum conditions. The double beam modulation technique, which was devised by M F A Harrison, has been described in reviews by Harrison [1,2] and
Table 1. $S$ represents signal counts, $N_{A}$ and $N_{B}$ are noise background counts due to beam A and beam B respectively (interacting with background gas/surfaces) and $\beta$ is the background noise when neither beam is present.

\begin{tabular}{llll}
\hline Channel & Beam A & Beam B & Counts \\
\hline 1 & ON & ON & $C_{1}=S+N_{a}+N_{B}+\beta$ \\
2 & ON & OFF & $C_{2}=N_{a}+\beta$ \\
3 & OFF & OFF & $C_{3}=\beta$ \\
4 & OFF & ON & $C_{4}=N_{B}+\beta$ \\
\hline
\end{tabular}

by Dolder [3]. This technique was first used by Dance et al [4] in 1965 , in a measurement of the cross section for electron impact excitation of the $1 \mathrm{~s}-2 \mathrm{~s}$ transition in $\mathrm{He}^{+}$.

Briefly, both beams are switched electronically as illustrated in figure 1, i.e. two equal duty cycle pulse trains with a $\pi / 2$ phase shift between them. This gives rise to four distinct beam configurations, or channels, 1 to 4 , per cycle, each with an associated noise rate as illustrated in table 1 .

Total signal counts after time $T$ are thus obtained by the algebraic sum of total counts $C_{1}$ to $C_{4}$ via

$$
S=C_{1}-C_{2}+C_{3}-C_{4} .
$$




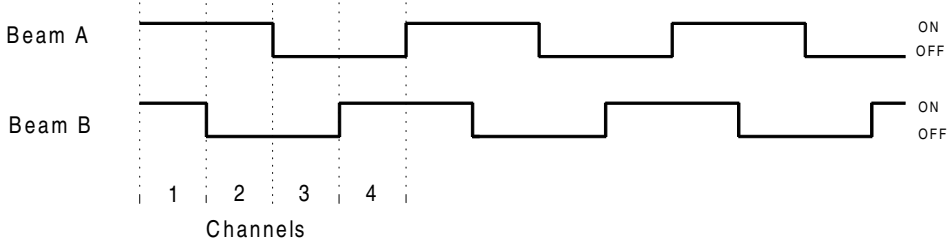

Figure 1. Standard double beam modulation pulse sequence, employing equal counting times in all four channels.

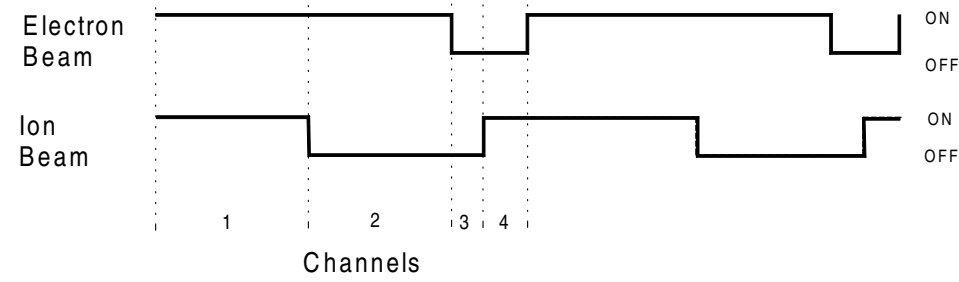

Figure 2. Time optimized modulation sequence based on sample count rates: $R_{1}=55000 \mathrm{~s}^{-1}, R_{2}=50000 \mathrm{~s}^{-1}, R_{3}=500 \mathrm{~s}^{-1}$, $R_{4}=5500 \mathrm{~s}^{-1}$. Then for a $1 \mathrm{~ms}$ modulation cycle $t_{1}=423 \mu \mathrm{s}, t_{2}=403 \mu \mathrm{s}, t_{3}=40 \mu \mathrm{s}$ and $t_{4}=134 \mu \mathrm{s}$.

In Harrison's original scheme two scalers are used; one scaler is gated to accumulate $C_{1}$ and $C_{3}$, while the second scaler is gated to accumulate $C_{2}$ and $C_{4}$. The signal is given by the difference in these two accumulated counts. Finesses in the implementation of this scheme included periodic phase reversal of both beam chopping cycles during acquisition to allow for any systematic variation in noise rates following switching of the beams. A periodic phase change was also introduced to effectively swap the roles of the two scalers, thus ensuring that any error due to counter differences was averaged out. Molyneux et al [5] have described an electronic unit for providing the necessary beam modulation and scaler gating pulses.

More recent implementations of the double beam modulation scheme have been carried out by Dunn's group at JILA [6], Chutjian's group at JPL [7] and in our own laboratory in Belfast [8]. These schemes place the beam switching sequence under computer control and allow counts from each of the four channels to be recorded separately. These schemes all use a $50 \%$ duty cycle in chopping the beams, and allow for restricted phase changes in the chopping sequence to minimize any effects due to subtle pressure or beam variation effects in the course of the modulation sequence.

The work reported here builds on the previous four channel schemes in allowing greater flexibility. The outstanding new feature we introduce is a variable duty cycle switching scheme (figure 2) that is specified so as to minimize the statistical uncertainty in the accumulated signal in a given time. The duty cycle requirements are calculated and implemented continuously in real time during an experimental run.

With accurate knowledge of the duty cycle the signal rate, $R_{S}$, measured in a time $T$, can be calculated as

$$
R_{S}=\frac{S}{\tau}=R_{1}-R_{2}+R_{3}-R_{4}
$$

The 'livetime' $\tau$ (time for both beams on) is slightly lower than the nominal $25 \%$ of $T$ due to a deadtime which allows the beams time to settle following each pulse edge and to avoid pick-up during the transient. The measured signal rate is also critically dependent on pulse deadtimes [8], but this effect will be ignored here. The modulation frequency is determined by the vacuum time constant of the experimental chamber. The modulation time period must be much shorter than the time during which an appreciable pressure change can occur in the apparatus. The vacuum time constant of the Belfast electron-ion apparatus is $\sim 100 \mathrm{~ms}$, and so we employ a beam modulation frequency of approximately $1 \mathrm{kHz}$.

In this communication we derive the condition necessary to minimize the statistical uncertainty obtaining in a given counting time. Further, we demonstrate a practical realization of this, which can lead to a $30 \%$ enhancement in efficiency for typical cases.

\section{Theory}

Consider a counting system operating for a single modulation time period of duration $T$. Let the rates in the four channels be denoted as $R_{i}, i=1$ to 4 , where $R_{i}=C_{i} / t_{i}$ and $t_{i}$ is the time for which counter $i$ is open. The aim is to minimize the statistical uncertainty in the measured signal rate $R_{S}$ in a given acquisition time $T$. To achieve this $t_{i}$, which were previously assumed constant $\left(t_{i}=T / 4\right)$, must be made variable as follows. The parameter to be minimized is the standard deviation in $R_{S}$

$$
\sigma_{R_{S}}=\left\{\sum_{i} \frac{R_{i}}{t_{i}}\right\}^{1 / 2}
$$

or, equivalently, the variance

$$
\sigma_{R_{s}}^{2}=\sum_{i} \frac{R_{i}}{t_{i}}
$$

subject to the constraint

$$
\sum_{i} t_{i}=T \quad \text { (constant). }
$$

Using the method of Lagrange multipliers we write

$$
F\left(t_{i}: i=1, \ldots, 4\right)=\sum_{i} \frac{R_{i}}{t_{i}}+\lambda \sum_{i} t_{i}
$$




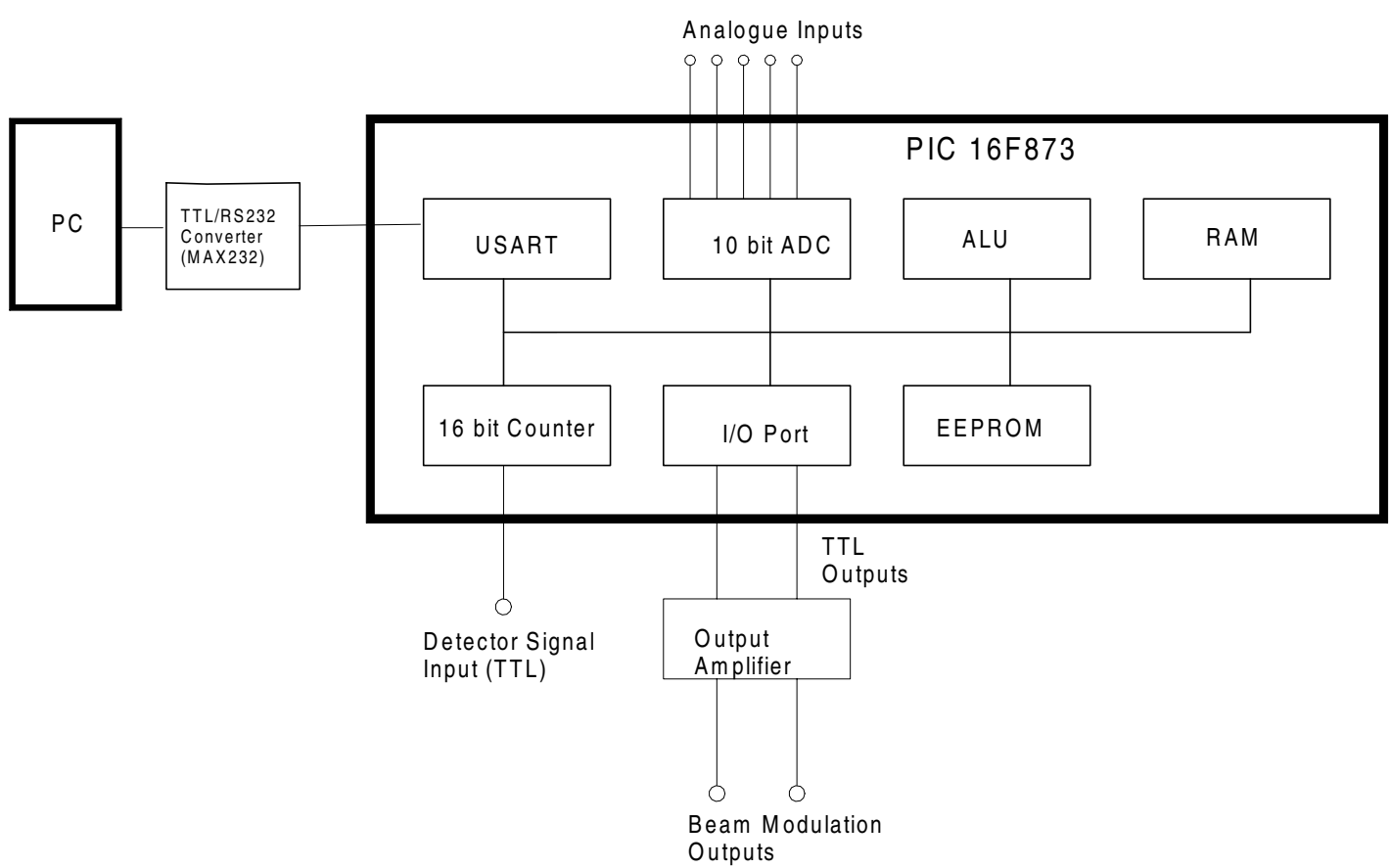

Figure 3. Schematic of microcontroller based modulation and counting unit. The entire unit is housed in a single width NIM unit. Operational parameters and data are transferred via a standard RS232 serial link to a PC workstation which carries out calculation of optimization parameters.

so that the minimization conditions $\partial F / \partial t_{i}=0$ give

$$
\lambda=\frac{R_{i}}{t_{i}^{o p t^{2}}}
$$

where $t_{i}^{\text {opt }}$ are the optimized acquisition times for each channel. Substituting for $t_{i}^{o p t}$ in (1) then gives

$$
\sum_{i} \sqrt{R_{i}}=T \sqrt{\lambda}
$$

so that the final optimization condition for the counting process is

$$
t_{i}^{o p t}=\frac{\sqrt{R_{i}} T}{\sum_{i} \sqrt{R_{i}}} .
$$

In this optimized case the variance in the measured signal rate $R_{S}$ after time $T_{o p t}$ is

$$
\sigma_{o p t}^{2}=\frac{1}{T_{o p t}}\left(\sum_{i} \sqrt{R_{i}}\right)^{2}
$$

whereas in the standard case of equal counting times in all four channels we have

$$
\sigma_{E Q}^{2}=\frac{4}{T_{E Q}} \sum_{i} R_{i} .
$$

Thus, the counting times required to attain a given level of statistical uncertainty in each case are related by

$$
\frac{T_{o p t}}{T_{E Q}}=\left(\sum_{i} \sqrt{R_{i}}\right)^{2}\left(4 \sum_{i} R_{i}\right)^{-1} \leqslant 1 .
$$

To see what practical advantage will accrue we use the example of electron impact excitation of $\mathrm{C}^{+}$ions undertaken at Belfast [8]. A beam of low energy electrons $(8-15 \mathrm{eV})$ intersects a beam of $5 \mathrm{kV} \mathrm{C}{ }^{+}$ions in a magnetically confined spectrometer under ultra-high vacuum conditions $(\sim 5 \times$ $10^{-10}$ mbar). Absolute cross-sections for excitation of the $\mathrm{C}^{+}$resonance transition $\left(2 \mathrm{~s}^{2} 2 \mathrm{p}^{2} \mathrm{P}-2 \mathrm{~s} 2 \mathrm{p}^{2}{ }^{2} \mathrm{D}\right)$ were measured using the electron energy loss technique; this involved counting electrons scattered with an energy loss of $9 \mathrm{eV}$. A trochoidal spectrometer $(\boldsymbol{E} \times \boldsymbol{B}$ field) was used to direct the entire scattered electron flux onto a channeltron detector. Typical inelastic scattering signal rates are about $10 \mathrm{~s}^{-1}$ compared to the noise rates listed in table 2 . These noise rates are typical of those obtained in similar previous measurements using the same apparatus in our laboratory.

The beam modulation waveforms required to implement this optimized acquisition scheme are illustrated in figure 2. The asymmetry of the waveforms depends entirely on the noise rates obtained in a particular measurement. Using the sample rates listed in table 2 in equation (5) gives

$$
\frac{T_{o p t}}{T_{E Q}}=0.69 \text {. }
$$

From equations (3) and (4) a standard deviation of $10 \%$ in the measurement of $R_{S}$ (i.e. approximately $\pm 1 \mathrm{~s}^{-1}$ ) may be achieved in times

$$
T_{E Q}=123.6 \mathrm{~h} \quad T_{o p t}=85.2 \mathrm{~h} .
$$

It is obvious that in an experiment of this kind, where the signal to noise ratio is very low, any improvement in counting efficiency will be important. Given the long acquisition times required a $30 \%$ reduction is a very considerable improvement. Such an improvement would be particularly helpful, for example, to experimentalists who are sharing run-time on a large multi-user accelerator facility. 
Table 2. Optimized channel widths $\left(t_{i} / T\right)$ for typical electron-ion noise rates.

\begin{tabular}{llllll}
\hline Channel $i$ & Electron & Ion & Noise $R_{i}\left(\mathrm{~s}^{-1}\right)$ & $\sqrt{R_{i}}\left(\sum_{i} \sqrt{R_{i}}\right)^{-1}$ & $t_{i} / T(\%)$ \\
\hline 1 & ON & ON & 55000 & 0.423 & 42.3 \\
2 & ON & OFF & 50000 & 0.403 & 40.3 \\
3 & OFF & OFF & 500 & 0.040 & 4.0 \\
4 & OFF & ON & 5500 & 0.134 & 13.4 \\
\hline
\end{tabular}

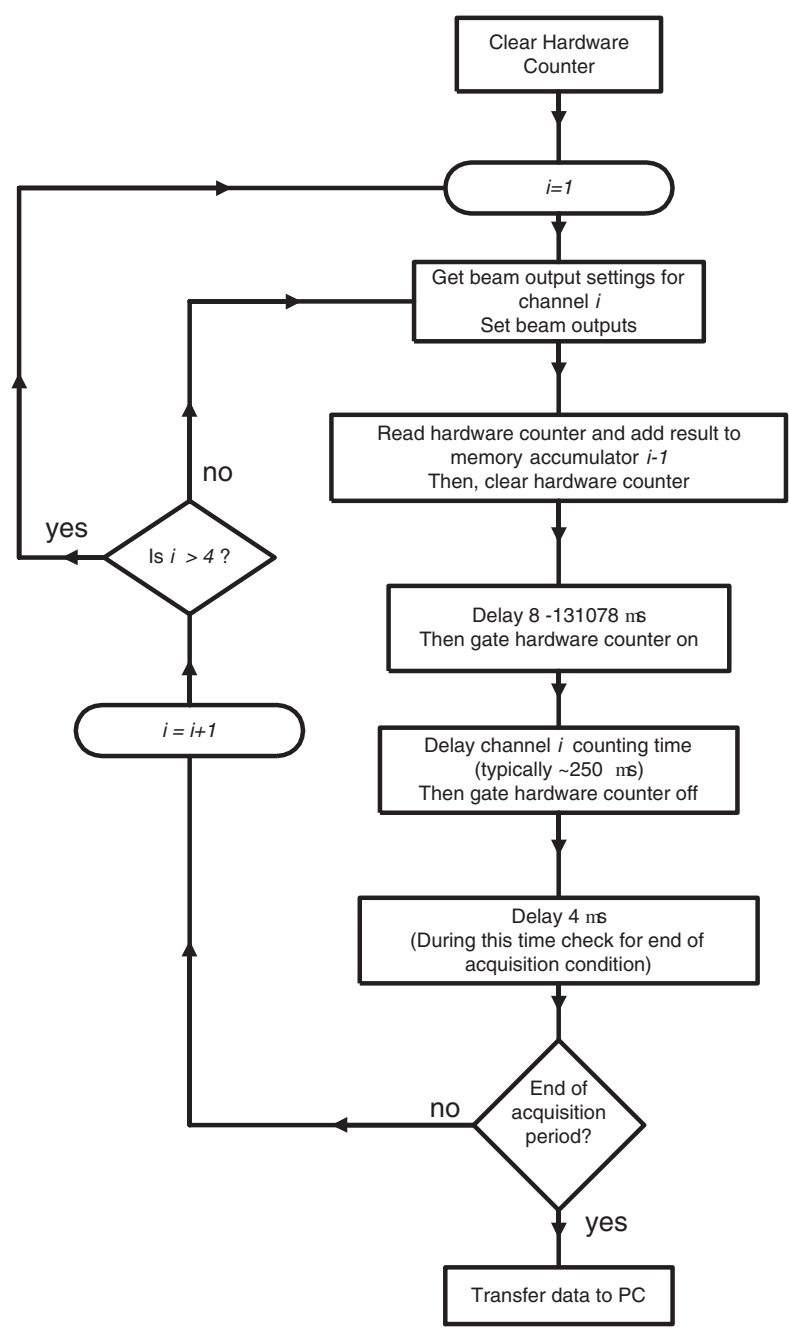

Figure 4. Simplified flow diagram of the modulation and counting control sequence.

\section{Microcontroller based modulation and counting system}

Realization of a real-time, optimized data acquisition system involves non-trivial technical requirements. To see this, consider the steps that will be needed in a given experimental measurement.

(1) The acquisition will begin with a measurement of the noise rates for each channel. This will be undertaken with equal channel widths.

(2) The noise rates obtained will be used to calculate the optimal channel widths according to the condition given in equation (2). Appropriate modulation waveforms must be produced and detector pulses must be counted in synchronization to these waveforms.

(3) Finally, the possibility that the beam setup and noise rates may drift over an extended run should be taken into account. If significant drift occurs the optimization condition will change and the channel widths should be modified accordingly.

To solve this problem we have designed and implemented a system based on a microcontroller (PIC 16F873 manufactured by Microchip Technology Inc. [9]) with a serial link to an old desktop 80486 PC running DOS. A master program on the PC (written in $\mathrm{C}++$ ) governs the overall acquisition; it provides a user interface, transfers data to and from the microcontroller, carries out the optimization calculations and stores data to disk. The microcontroller controls the modulation of the beams and carries out event counting in synchronization with these modulation waveforms.

The beam modulation and counting system requires one input for conditioned detector pulses to be counted (TTL), and two beam switching outputs $(0-10 \mathrm{~V})$. These switching outputs drive external push-pull amplifier circuitry that is used to provide high voltage levels required to directly manipulate the experimental beams (electrostatic deflection). This system has been implemented as a NIM unit for convenience, with an RS232 port to allow serial data transfer to and from the controlling PC at baud rates up to $56 \mathrm{~K}$. This system is shown schematically in figure 3 .

The PIC16F873 microcontroller offers a versatile combination of hardware and software flexibility. This low cost device (less than \$10) features $16 \mathrm{I} / \mathrm{O}$ pins, one 16 bit counter, two eight bit counters, one 10 bit ADC (with up to five analogue inputs) and serial data transfer capability. On chip memory comprises $4 \mathrm{~K} \times 14$ words of program memory (ROM), 192 bytes of RAM and 128 bytes of EEPROM data memory. The device operates at $20 \mathrm{MHz}$ clock speed, with an instruction cycle of $200 \mathrm{~ns}$. All instructions are single cycle except for instructions that change flow of execution, which are always two cycle. As a result all timing may be controlled with nanosecond accuracy (limited by clock stability) simply by counting software instruction cycles, although the timing resolution is limited by, and equal to, the instruction cycle time of $200 \mathrm{~ns}$.

The acquisition cycle is controlled by instructions held in ROM on the PIC chip. Two digital outputs are used to provide the beam modulation waveforms and all detector pulse counting is carried out by the single 16 bit hardware counter. The essential steps in the microcontroller software sequence are shown in figure 4. (This is slightly simplified in the interests of clarity.) Four 32 bit memory locations are used to accumulate the counts for each channel of the modulation sequence. Another further software accumulator (not indicated in figure 4) keeps track of the number of modulation cycles that have elapsed. At the end of a predetermined number 


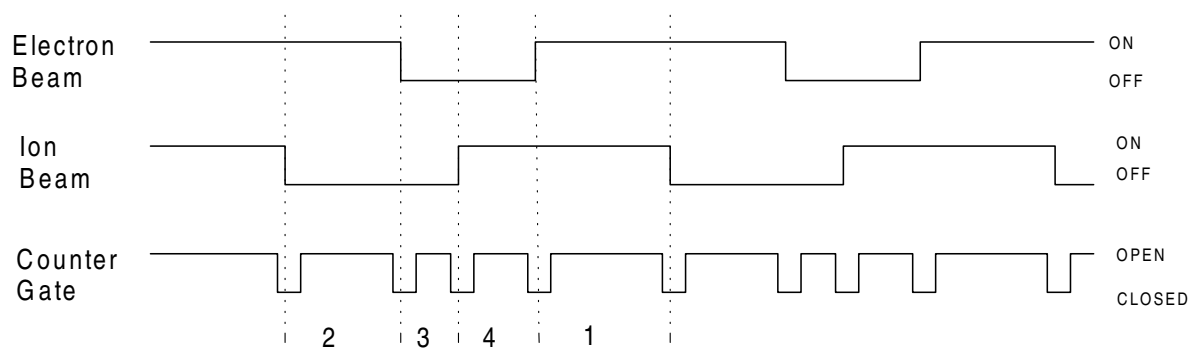

Figure 5. Synchronized modulation and counter gating sequence. The sample channel widths shown are $t_{1}=350 \mu \mathrm{s}, t_{2}=300 \mu \mathrm{s}$, $t_{3}=150 \mu \mathrm{s}$ and $t_{4}=200 \mu \mathrm{s}$. There is a non-counting period (typically $20 \mu \mathrm{s}$ ) around each beam transition to allow for transients and settling.
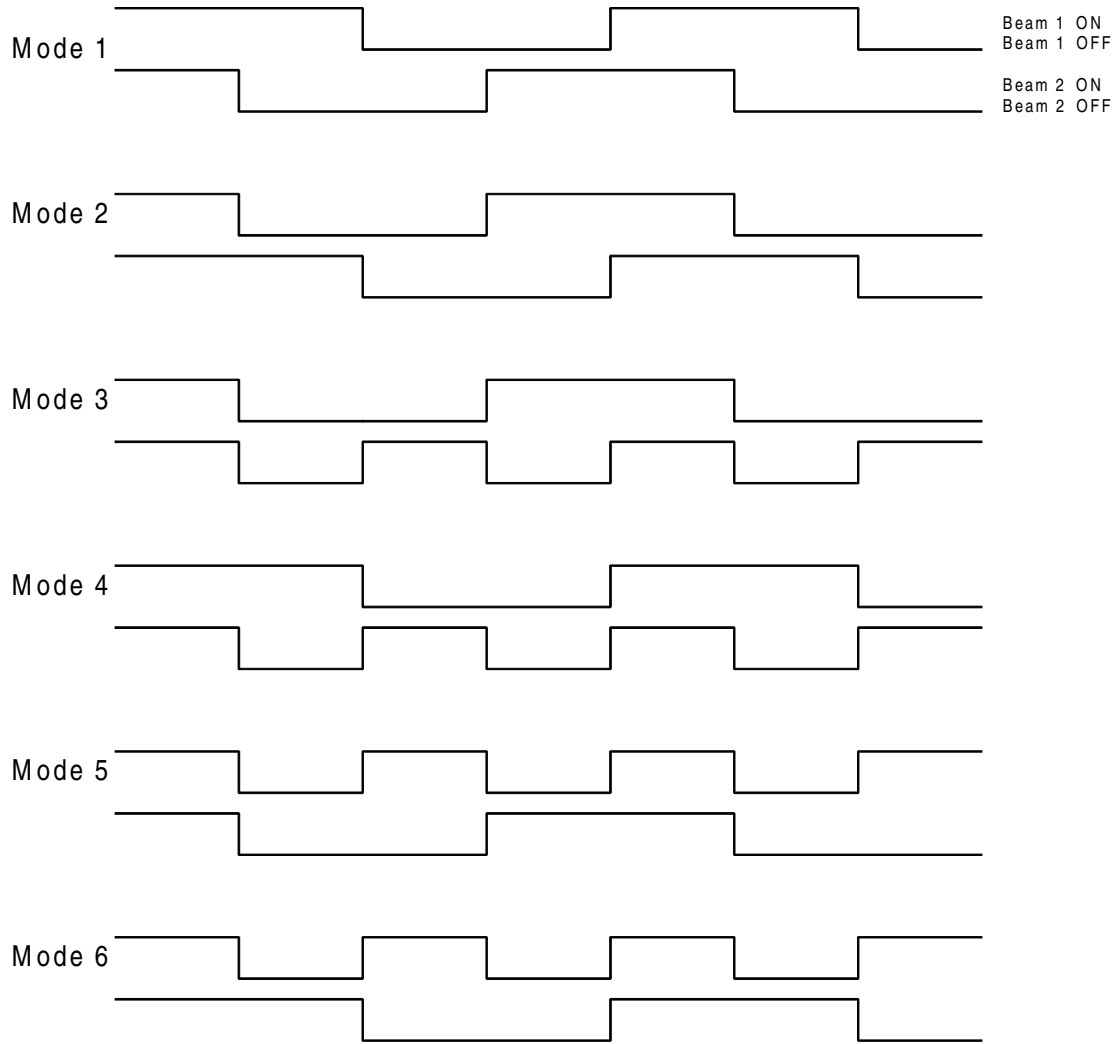

Figure 6. Distinct beam modulation modes. These are displayed with equal channel time widths for convenience. Data acquisition employs each of these modes in rotation to negate any possible signal modulation effects that are order dependent.

of cycles data are transferred to the controlling PC via the serial link. A typical acquisition schedule is as follows: the acquisition period between transfers to the $\mathrm{PC}$ is $1.08 \mathrm{~s}$. This comprises 1000 modulation cycles. For each cycle the four channel counting times add up to exactly $1000 \mu \mathrm{s}$, and there is a non-counting deadtime of $4 \mu$ s before and $16 \mu$ s after every beam switching event (see figure 5).

A more detailed exposition of the microcontroller operation may be valuable in order to fully underscore the flexibility of this system. For each acquisition period the following steps are carried out.

(1) Set the beam modulation outputs: the modulation sequence is specified by the bit pattern stored in a specific byte in RAM. Pairs of bits represent each setting for the beam output states. For example, the bit pattern 00011011 specifies the sequence of channels $(1,2,4,3)$.
(2) Delay for a specified time to allow beams to settle after switching (this is programmable in the range 8-131078 $\mu \mathrm{s})$. During this time two additional operations are performed:

(a) the value from hardware counter is added to the software accumulator corresponding to the previous channel (modulo 4) and

(b) the hardware counter is cleared.

(3) Start the counting process (open counter gate).

(4) Wait for the required channel acquisition time to elapse, then stop counting. This time has been calculated based on the previously measured noise rates for each channel and stored in microcontroller RAM. The range of acceptable values is 4-131074 $\mu$ s (in $2 \mu$ s steps).

(5) For the fourth channel in a cycle the program updates the software cycle counter and checks to see whether 
the required number of cycles have elapsed, i.e., the acquisition period is completed. If so, the hardware counter is read and the final channel accumulator is updated. If the acquisition period is incomplete, the program delays for $4 \mu$ s then branches to step (1). Step (5) always takes exactly $4 \mu$ s to execute regardless of channel number.

While this basic acquisition sequence remains fixed, almost every parameter of the acquisition can be controlled by appropriate data transfer to the microcontroller. Most important of these are the individual channel timings. Once the noise rates have been measured and correct channel widths calculated on the controlling PC according to the optimization algorithm, these timings are then transferred to PIC RAM. If noise rates vary in the course of acquisition it is a simple matter to track these and adjust the beam modulation scheme as required. There is complete control of the overall modulation frequency.

The non-counting time around each beam transition is also programmable. This dead time is necessary to permit settling of the beams after deflection, and also to ensure that no electronic pick-up due to the beam switching affects the count process. While efficiency requires as short a dead time as possible, a compromise is inevitable. The dead time is programmable in the range 12-131082 $\mu$ s.

Of considerable importance is the fact that the beam modulation sequence is completely programmable. There are six modes of operation (distinct orderings) possible in the modulation cycle (shown in figure 6) and each of these may be implemented as desired. Changing the modulation sequence and acquiring data using all possible sequences guards against the possibility that the small signal we seek to measure may depend in some unexpected way on the order in which the beams are switched. By averaging over all possible switching sequences such an effect may be minimized. Moreover, by sweeping the dead time, the same device can be used to look for such effects in boxcar-like mode. It is also worth noting that, since only one hardware counter is used for all four modulation channels, there is no possibility of small differences in the operation of multiple counters leading to spurious results.

Typically we operate at a beam modulation frequency of slightly less than $1 \mathrm{kHz}$ ( $1 \mathrm{~ms}$ livetime plus $20 \mu$ s deadtime at each beam switch). Data transfer after each 1000 cycles allows updating of the measured signal approximately once per second. A new mode of operation (channel order) is selected every 100 seconds. Typically data transfer and updating lead to a total acquisition deadtime of approximately $10 \%$ at $1 \mathrm{kHz}$. This is regarded as acceptable.

\section{Conclusions}

We have developed a microcontroller based double beam modulation and counting system for atomic scattering experiments. We are sure that the flexibility and ease of use of the microcontroller means that such devices will find widespread application in atomic physics research laboratories, and we recommend them to other researchers. Complete software control of the acquisition process has enabled us to implement an optimization procedure that provides significant reductions in counting time for time consuming electron-ion scattering experiments.

\section{Acknowledgments}

We are grateful to Drs B Peart and C J Latimer for discussions that stimulated us to investigate the optimization of our counting system. We acknowledge financial support from EPSRC (UK) (IDW, JBG) and from Enterprise Ireland/British Council Research Visits Grants scheme (RWO’N, IDW).

\section{References}

[1] Harrison M F A 1966 Br. J. Appl. Phys. 17371

[2] Harrison M F A 1968 Methods in Experimental Physics vol 7A, ed B Bederson and W L Fite (New York: Academic)

[3] Dolder K T 1969 Case Studies in Atomic Collision Physics vol 1, ed E W McDaniel and M R C McDowell (Amsterdam: North-Holland) p 249

[4] Dance D F, Harrison M F A and Smith A C H 1966 Proc. $R$. Soc. A 29073

[5] Molyneux L, Dolder K T and Peart B 1971 J. Phys. E: Sci. Instrum. 4149

[6] Bell E W et al 1994 Phys. Rev. A 494585

[7] Smith S J et al 1993 Phys. Rev. A 48292

[8] Williams I D, Greenwood J B, Srigengan B, O’Neill R W and Hughes I G 1998 Meas. Sci. Technol. 9930

[9] Microchip Technology Inc., USA 2000 PIC 16F873 Datasheet www.microchip.com 